\title{
Emergent Inert Adjoint Scalar Field in SU(2) Yang-Mills Thermodynamics due to Coarse-Grained Topological Fluctuations
}

\author{
Ulrich Herbst ${ }^{1}$ and Ralf Hofmann ${ }^{2}$ \\ ${ }^{1}$ Institut für Theoretische Physik, Universität Heidelberg, Philosophenweg 16, 69120 Heidelberg, Germany \\ ${ }^{2}$ Institut für Theoretische Physik, Universität Frankfurt, Johann Wolfgang Goethe-Universität, \\ Max von Laue-Straße 1, 60438 Frankfurt, Germany
}

Correspondence should be addressed to Ralf Hofmann, r.hofmann@thphys.uni-heidelberg.de

Received 3 November 2011; Accepted 6 December 2011

Academic Editor: A. Belhaj

Copyright (C) 2012 U. Herbst and R. Hofmann. This is an open access article distributed under the Creative Commons Attribution License, which permits unrestricted use, distribution, and reproduction in any medium, provided the original work is properly cited.

\begin{abstract}
We compute the phase and the modulus of an energy- and pressure-free, composite, adjoint, and inert field $\phi$ in an SU(2) Yang-Mills theory at large temperatures. This field is physically relevant in describing part of the ground-state structure and the quasiparticle masses of excitations. The field $\phi$ possesses nontrivial $S^{1}$-winding on the group manifold $S^{3}$. Even at asymptotically high temperatures, where the theory reaches its Stefan-Boltzmann limit, the field $\phi$, though strongly power suppressed, is conceptually relevant: its presence resolves the infrared problem of thermal perturbation theory.
\end{abstract}

\section{Introduction}

In $[1,2]$ one of us has put forward an analytical and nonperturbative approach to $\mathrm{SU}(2) / \mathrm{SU}(3)$ Yang-Mills thermodynamics. Each of these theories comes in three phases: deconfining (electric phase), preconfining (magnetic phase), and completely confining (center phase). This approach assumes the existence of a composite, adjoint Higgs field $\phi$, describing part of the thermal ground state, that is, the BPS saturated topologically nontrivial sector of the theory. The field $\phi$ is generated by a spatial average over noninteracting trivial-holonomy SU(2) calorons [3] which can be embedded in SU(3). The "condensation"1 of trivial-holonomy $\mathrm{SU}(2)$ calorons into the field $\phi$ must take place at an asymptotically high temperature $[1,2]$, that is, at the limit of applicability of the gauge-field theoretical description. For any physics model formulated in terms of an SU(2)/SU(3) Yang-Mills theory this is to say that caloron "condensation" takes place at $T \sim M_{P}$ where $M_{P}$ denotes 
the Planck mass. Since $|\phi| \sim \sqrt{\Lambda^{3} / 2 \pi T}$ topological defects only marginally deform the idealgas expressions for thermodynamical quantities at $T \gg \Lambda$. Here $\Lambda$ denotes the Yang-Mills scale. Every contribution to a thermodynamical quantity, which arises from the topologically nontrivial sector, is power suppressed in temperature. As a consequence, the effective theory is asymptotically free and exhibits, though in a quantitatively different way, the infraredultraviolet decoupling property [1,2] seen in renormalized perturbation theory [4-7]. Asymptotic freedom is a conceptually very appealing property of $\mathrm{SU}(N)$ Yang-Mills theories. It first was discovered in perturbation theory [8-11].

In the effective thermal theory, interactions between trivial-holonomy calorons in the ground state are taken into account by obtaining a pure-gauge solution to the classical equations of motion for the topologically trivial sector in the (nonfluctuating and nonbackreacting) background $\phi$. Thus the partition function of the fundamental theory is evaluated in three steps in the deconfining phase: (i) integrate over the admissible part of the moduli space for the caloron-anticaloron system and spatially average over the associated two-point correlations to derive the (classical and temperature dependent) dynamics of an adjoint, spatially homogeneous scalar field $\phi$, (ii) establish the quantum mechanical and statistical inertness of $\phi$ and use it as a temperature-dependent background to find a pure-gauge solution $a_{\mu}^{\mathrm{bg}}$ to the Yang-Mills equations describing the trivial-topology sector. Together, $\phi$ and $a_{\mu}^{\mathrm{bg}}$ constitute the thermal ground state of the system. The fact that the action for the ground-state configurations $\phi$ and $a_{\mu}^{\mathrm{bg}}$ is infinite is unproblematic since the corresponding, vanishing weight in the partition function is associated with a nonfluctuating configuration and therefore can be factored out and is cancelled when evaluating expectation values in the effective theory. (iii) Consider the interactions between the macroscopic ground state and trivial-topology fluctuations in terms of quasiparticle masses of the latter which are generated by the adjoint Higgs mechanism ${ }^{2}$ and impose thermodynamical self-consistency to derive an evolution equation for the effective gauge coupling $e$. In the following, we will restrict our discussion to the case $\mathrm{SU}(2)$. Isolated magnetic charges are generated by dissociating calorons of sufficiently large holonomy [12-23]; for a quick explanation of the term holonomy, see Figure 1. Nontrivial holonomy is locally excited by interactions between trivial-holonomy calorons and anticalorons mediated by plane-wave configurations. In [18] it was shown as a result of a heroic calculation that small (large) holonomy leads to an attractive (repulsive) potential between the monopole and the antimonopole constituents of a given caloron. An attraction between a monopole and an antimonopole leads to annihilation once the distance between their centers is comparable to the sum of their charge radii. Thermodynamically, the probability for exciting a small holonomy is much larger than that for exciting a large holonomy. In the former case, this probability roughly is determined by the one-loop quantum weight of a trivial holonomy caloron, while in the latter case both monopole constituents have a combined mass $\sim 4 \pi^{2} T \sim 39 T$ [14]. Thus an attractive potential between a monopole and its antimonopole is the by far dominating situation. This is the microscopic reason for a negative ground-state pressure $P^{\text {g.s. }}$ which, on spatial average, turns out to be $P^{\text {g.s. }}=-4 \pi \Lambda^{3} T$ (the equation of ground state is $\rho^{\text {g.s. }}=-P^{\text {g.s. }}$ ) $[1,2]$. In the unlikely case of repulsion (large holonomy) the monopole and the antimonopole separate back to back until their interaction is sufficiently screened to facilitate their existence in isolation (as long as the overall pressure of the system is positive). Magnetic monopoles in isolation do not contribute to the pressure of the system ${ }^{3}$. The overall pressure is positive if the gauge-field fluctuations after spatial coars graining are sufficiently light and energetic to over compensate the negative ground-state contribution, that is, if the temperature is sufficiently large. 


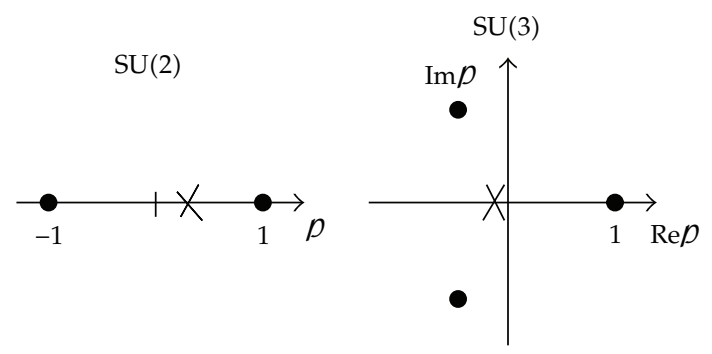

Figure 1: Possible values of the Polyakov loop $D$ at spatial infinity on a given gauge-field configuration. A small holonomy corresponds to values close to the center elements depicted by the dots. Crosses indicate examples for a large holonomy.

Caloron-induced tree-level masses for gauge-field modes decay as $1 / \sqrt{T}$ when heating up the system. Due to the linear rise of $\rho^{\text {g.s. }}$ with $T$ the thermodynamics of the ground state is thus subdominant at large temperatures ${ }^{4}$. The main purpose of the present work is to compute and to discuss the dynamical generation of an adjoint, macroscopic, and composite scalar field $\phi$. This is a first-principle analysis of the ground-state structure in the electric phase of an SU(2) Yang-Mills theory. The paper is organized as follows. In Section 2 we write down and discuss a nonlocal definition, relevant for the determination of $\phi^{\prime}$ s phase, in terms of a spatial and scale-parameter average over an adjointly transforming 2-point function. This average needs to be evaluated on trivial-holonomy caloron and anticaloron configurations at a given time $\tau$. In Section 3 we perform the average and discuss the occurrence of a global gauge freedom in $\phi^{\prime}$ s phase, which has a geometrical interpretation. In Section 4 , we show how the derived information about a nontrivial $S^{1}$ winding of the field $\phi$ together with analyticity of the right-hand side of the associated BPS equation and with the assumption of the existence of an externally given scale $\Lambda$ can be used to uniquely construct a potential determining $\phi$ 's classical (and temperature dependent) dynamics. In Section 5 we summarize and discuss our results and give an outlook on future research.

\section{Definition of $\phi^{\prime}$ s Phase}

In this section we discuss the BPS saturated, topological part of the ground-state physics in the electric phase of an SU(2) Yang-Mills theory. According to the approach in $[1,2]$ the adjoint scalar $\phi$ emerges as an energy- and pressure-free (BPS saturated) field from a spatial average over the classical correlations in a caloron-anticaloron system of trivial holonomy in absence of interactions. On spatial average, the latter are taken into account by a pure-gauge configuration solving the classical, trivial-topology gauge-field equations in the spatially homogeneous background $\phi$. This is consistent since $\phi$ 's quantum mechanical and statistical inertness can be established. Without assuming the existence of a Yang-Mills scale $\Lambda$ only $\phi^{\prime}$ 's phase, that is $\phi /|\phi|$, can be computed. A computation of $\phi$ itself requires the existence of $\Lambda$. As we will see, the information about the $S^{1}$ winding of $\phi^{\prime}$ s phase together with the analyticity of the right-hand side of $\phi^{\prime}$ s BPS equation uniquely determines $\phi^{\prime}$ 's modulus in terms of $\Lambda$ and $T$.

Let us first set up some prerequisites. We consider BPS saturated solutions to the YangMills equation

$$
D_{\mu} F_{\mu \nu}=0
$$


which are periodic in Euclidean time $\tau(0 \leq \tau \leq 1 / T)$ and of trivial holonomy. The only relevant configurations are calorons of topological charge $e^{5} \pm 1$. They are [3]

$$
\begin{aligned}
& A_{\mu}^{\mathrm{C}}(\tau, \mathbf{x})=\bar{\eta}_{a \mu \nu} \frac{\lambda^{a}}{2} \partial_{\nu} \ln \Pi(\tau, \mathbf{x}) \text { or } \\
& A_{\mu}^{A}(\tau, \mathbf{x})=\eta_{a \mu \nu} \frac{\lambda^{a}}{2} \partial_{\nu} \ln \Pi(\tau, \mathbf{x}),
\end{aligned}
$$

where the 't Hooft symbols $\eta_{a \mu \nu}$ and $\bar{\eta}_{a \mu \nu}$ are defined as

$$
\begin{aligned}
& \eta_{a \mu \nu}=\epsilon_{a \mu \nu}+\delta_{a \mu} \delta_{v 4}-\delta_{a v} \delta_{\mu 4} \\
& \bar{\eta}_{a \mu \nu}=\epsilon_{a \mu \nu}-\delta_{a \mu} \delta_{v 4}+\delta_{a v} \delta_{\mu 4} .
\end{aligned}
$$

The solutions in (2.2) (the superscript (A)C refers to (anti)caloron) are generated by a temporal mirror sum of the (pre) potential $\Pi$ of a single (anti)instanton in singular gauge [2426]. They have the same color orientation as the "seed" instanton or "seed" anti-instanton. In (2.2) $\lambda^{a},(a=1,2,3)$, denote the Pauli matrices. The "nonperturbative" definition of the gauge field is used were the gauge coupling constant $g$ is absorbed into the field.

The scalar function $\Pi(\tau, \mathbf{x})$ is given as [3]

$$
\Pi(\tau, \mathbf{x})=\Pi(\tau, r) \equiv 1+\frac{\pi \rho^{2}}{\beta r} \frac{\sinh (2 \pi r / \beta)}{\cosh (2 \pi r / \beta)-\cos (2 \pi \tau / \beta)}
$$

where $r \equiv|\mathbf{x}|, \beta \equiv 1 / T$, and $\rho$ denotes the scale parameter whose variation leaves the classical action $S=8 \pi^{2} / g^{2}$ invariant. At a given $\rho$ the solutions in (2.2) can be generalized by shifting the center from $z=0$ to $z=\left(\tau_{z}, \mathbf{z}\right)$ by the (quasi)translational invariance of the classical action $^{6} S$. Another set of moduli is associated with global color rotations of the solutions in (2.2).

From the BPS saturation

$$
F_{\mu \nu}\left[A^{(C, A)}\right]=(+,-) \widetilde{F}_{\mu \nu}\left[A^{(C, A)}\right]
$$

it follows that the (Euclidean) energy-momentum tensor $\theta_{\mu \nu}$, evaluated on $A_{\mu}^{(C, A)}$, vanishes identically

$$
\theta_{\mu \nu}\left[A^{(C, A)}\right] \equiv 0
$$

This property translates to the macroscopic field $\phi$ with energy-momentum tensor $\bar{\theta}_{\mu \nu}$ in an effective theory since $\phi$ is obtained by a spatial average over caloron-anticaloron correlations neglecting their interactions ${ }^{7}$

$$
\bar{\theta}_{\mu v}[\phi] \equiv 0
$$




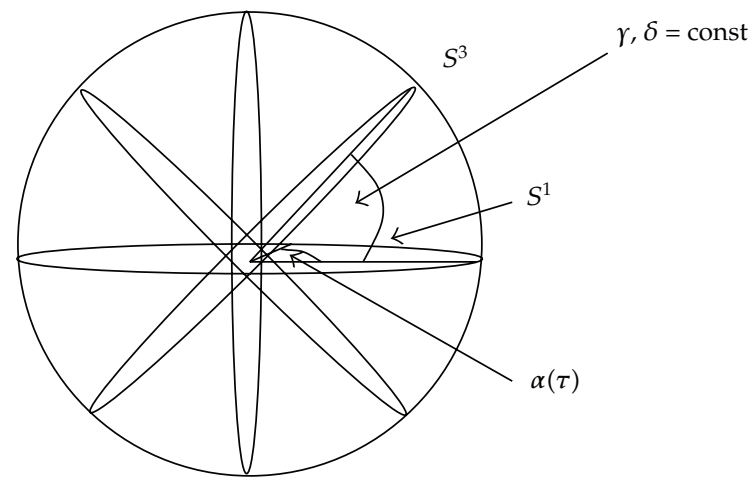

Figure 2: Possible directions of winding of $(\phi /|\phi|)(\tau)$ around the group manifold $S^{3}$ of SU(2). The angles $\gamma, \delta$ are arbitrary but constant. They are determined by the choice of plane in which angular regularization is carried out when computing $\phi^{\prime}$ s phase, see below. The angle $\alpha(\tau)$ parametrizes the $S^{1}$ winding of $\phi /|\phi|$.

The field $\phi$ is spatially homogeneous since it emerges from a spatial average. If the action density governing $\phi^{\prime}$ s dynamics in the absence of caloron interactions contains a kinetic term quadratic in the $\tau$-derivatives and a potential $V$ then (2.7) is equivalent to $\phi$ solving the firstorder equation

$$
\partial_{\tau} \phi=V^{(1 / 2)}
$$

where $V^{(1 / 2)}$ denotes the "square $\operatorname{root}^{\text {" of }} V^{8}, V \equiv \operatorname{tr}\left(V^{(1 / 2)}\right)^{\dagger} V^{(1 / 2)}$. In (2.8) the right-hand side will turn out to be determined only up to a global gauge rotation, see Figure 2. Already at this point it is important to remark that the Yang-Mills scale parametrizes the potential $V$ and thus also the classical solution to (2.8). In the absence of trivial-topology fluctuations it is, however, invisible, see (2.7). Only after the macroscopic equation of motion for the trivial-topology sector is solved for a pure-gauge configuration in the background $\phi$ does the existence of a Yang-Mills scale become manifest by a nonvanishing ground-state pressure and a nonvanishing ground-state energy density $[1,2]$. Hence the trace anomaly $\tilde{\theta}_{\mu \mu} \neq 0$ for the total energy-momentum tensor $\tilde{\theta}_{\mu \nu} \equiv \bar{\theta}_{\mu \nu}^{\text {g.s. }}+\theta_{\mu \nu}^{\text {fluc }}$ in the effective theory which includes the effects of trivial-topology fluctuations: since $\bar{\theta}_{\mu \nu}^{\text {g.s. }}=4 \pi T \Lambda^{3} \delta_{\mu \nu}$ and $\theta_{\mu \nu}^{\text {fluc }} \alpha T^{4}$ for $T \gg \Lambda$ the trace anomaly dies off as $\Lambda^{3} / T^{3}$.

Without imposing constraints other than nonlocality ${ }^{9}$ the $\tau$ dependence of $\phi^{\prime}$ s phase (the ratio of the two averages $\phi$ and $|\phi|$ over admissible moduli deformations, $A_{\mu}^{(C, A)}$ would naively be characterized as

$$
\begin{aligned}
\frac{\phi^{a}}{|\phi|}(\tau) \sim \operatorname{tr}\left[\beta^{0} 1 ! \int d^{3} x \int d \rho\right. \\
\quad \frac{\lambda^{a}}{2} F_{\mu \nu}\left[A_{\alpha}(\rho, \beta)\right]((\tau, 0))\{(\tau, 0),(\tau, \mathbf{x})\}\left[A_{\alpha}(\rho, \beta)\right] \\
\quad \times F_{\mu \nu}\left[A_{\alpha}(\rho, \beta)\right]((\tau, \mathbf{x}))\{(\tau, \mathbf{x}),(\tau, 0)\}\left[A_{\alpha}(\rho, \beta)\right] \\
+\beta^{-1} 2 ! \int d^{3} x \int d^{3} y \int d \rho
\end{aligned}
$$




$$
\begin{aligned}
& \frac{\lambda^{a}}{2} F_{\mu \lambda}\left[A_{\alpha}(\rho, \beta)\right]((\tau, 0))\{(\tau, 0),(\tau, \mathbf{x})\}\left[A_{\alpha}(\rho, \beta)\right] \\
& \times F_{\lambda \nu}\left[A_{\alpha}(\rho, \beta)\right]((\tau, \mathbf{x}))\{(\tau, \mathbf{x}),(\tau, \mathbf{y})\}\left[A_{\alpha}(\rho, \beta)\right] \\
& \times F_{\nu \mu}\left[A_{\alpha}(\rho, \beta)\right]((\tau, \mathbf{y}))\{(\tau, \mathbf{y}),(\tau, 0)\} \\
& +\beta^{-2} 3 ! \int d^{3} x \int d^{3} y \int d^{3} u \int d \rho \\
& \frac{\lambda^{a}}{2} F_{\mu \lambda}\left[A_{\alpha}(\rho, \beta)\right]((\tau, 0))\{(\tau, 0),(\tau, \mathbf{x})\}\left[A_{\alpha}(\rho, \beta)\right] \\
& \times F_{\lambda v}\left[A_{\alpha}(\rho, \beta)\right]((\tau, \mathbf{x}))\{(\tau, \mathbf{x}),(\tau, \mathbf{y})\}\left[A_{\alpha}(\rho, \beta)\right] \\
& \times F_{\nu \kappa}\left[A_{\alpha}(\rho, \beta)\right]((\tau, \mathbf{y}))\{(\tau, \mathbf{y}),(\tau, \mathbf{u})\} F_{\kappa \mu}\left[A_{\alpha}(\rho, \beta)\right]((\tau, \mathbf{u})) \\
& \times\{(\tau, \mathbf{u}),(\tau, 0)\}+\cdots] .
\end{aligned}
$$

The dots in (2.9) stand for the contributions of higher $n$-point functions and for reducible, that is, factorizable, contributions with respect to the spatial integrations. In (2.9) the following definitions apply:

$$
\begin{gathered}
|\phi| \equiv \frac{1}{2} \operatorname{tr} \phi^{2} \\
\{(\tau, 0),(\tau, \mathbf{x})\}\left[A_{\alpha}\right] \equiv p \exp \left[i \int_{(\tau, 0)}^{(\tau, \mathbf{x})} d y_{\beta} A_{\beta}(y, \rho)\right] \\
\{(\tau, \mathbf{x}),(\tau, 0)\}\left[A_{\alpha}\right] \equiv p \exp \left[-i \int_{(\tau, 0)}^{(\tau, \mathbf{x})} d y_{\beta} A_{\beta}(y, \rho)\right] .
\end{gathered}
$$

The integral in the Wilson lines in (2.10) is along a straight line ${ }^{10}$ connecting the points $(\tau, 0)$ and $(\tau, \mathbf{x})$, and $\boldsymbol{D}$ denotes the path-ordering symbol.

Under a microscopic gauge transformation $\Omega(y)$ the following relations hold:

$$
\begin{aligned}
\{(\tau, 0),(\tau, \mathbf{x})\}\left[A_{\alpha}\right] & \longrightarrow \Omega^{\dagger}((\tau, 0))\{(\tau, 0),(\tau, \mathbf{x})\}\left[A_{\alpha}\right] \Omega((\tau, \mathbf{x})) \\
\{(\tau, \mathbf{x}),(\tau, 0)\}\left[A_{\alpha}\right] & \longrightarrow \Omega^{\dagger}((\tau, \mathbf{x}))\{(\tau, \mathbf{x}),(\tau, 0)\}\left[A_{\alpha}\right] \Omega((\tau, 0)), \\
F_{\mu \nu}\left[A_{\alpha}\right]((\tau, \mathbf{x})) & \longrightarrow \Omega^{\dagger}((\tau, \mathbf{x})) F_{\mu \nu}\left[A_{\alpha}\right]((\tau, \mathbf{x})) \Omega((\tau, \mathbf{x})), \\
F_{\mu \nu}\left[A_{\alpha}\right]((\tau, 0)) & \longrightarrow \Omega^{\dagger}((\tau, 0)) F_{\mu \nu}\left[A_{\alpha}\right]((\tau, 0)) \Omega((\tau, 0)) .
\end{aligned}
$$

As a consequence of (2.11) the right-hand side of (2.9) transforms as

$$
\frac{\phi^{a}}{|\phi|}(\tau) \longrightarrow R_{a b}(\tau) \frac{\phi^{b}}{|\phi|}(\tau)
$$


where the $\mathrm{SO}(3)$ matrix $R_{a b}(\tau)$ is defined as

$$
R^{a b}(\tau) \lambda^{b}=\Omega((\tau, 0)) \lambda^{a} \Omega^{\dagger}((\tau, 0))
$$

Thus we have defined an adjointly transforming scalar in (2.9). Moreover, only the timedependent part of a microscopic gauge transformation survives after spatial averaging (macroscopic level).

In (2.9) the $\sim$ sign indicates that both left- and right-hand sides satisfy the same homogeneous evolution equation in $\tau$

$$
\Phi\left[\frac{\phi}{|\phi|}\right]=0
$$

Here $\Phi$ is a differential operator such that (2.14) represents a homogeneous differential equation. As it will turn out, (2.14) is a linear second-order equation which, up to global gauge rotations, determines the first-order or BPS equation whose solution $\phi$ 's phase is. Each term in the series in (2.9) is understood as a sum over the two solutions in (2.2), that is, $A_{\alpha}=A_{\alpha}^{C}$ or $A_{\alpha}=A_{\alpha}^{A}$. As we will show in Section 3, the dimensionless quantity defined on the right-hand side of (2.9) is ambiguous ${ }^{11}$; the operator $\Phi$, however, is not.

The quantities appearing in the numerator and denominator of the left-hand side of (2.9) are understood as functional and spatial averages over the appropriate multilocal operators, being built of the field strength and the gauge field. The functional average is restricted to the moduli spaces of $A_{\alpha}=A_{\alpha}^{C}$ and $A_{\alpha}=A_{\alpha}^{A}$ excluding global color rotations and time translations.

Let us explain this in more detail. For the gauge variant density in (2.9) an average over global color rotations and time shifts $\tau \rightarrow \tau+\tau_{z}\left(0 \leq \tau_{z} \leq \beta\right)$ would yield zero and thus is forbidden ${ }^{12}$. The nonflatness of the measure with respect to the separate $\rho$ integration in the numerator and the denominator average in (2.9) transforms into flatness by taking the ratio. Since the integration weight $\exp (-S)$ is independent of temperature on the moduli space of a caloron, the right-hand side of (2.9) must not exhibit an explicit temperature dependence. This forbids the contribution of $n$-point functions with $n>2$, and we are left with an investigation of the first term in (2.9). In the absence of a fixed mass scale on the classical level an average overspatial translations would have a dimensionful measure $d^{3} z$ making the definition of a dimensionless quantity $\sim \phi /|\phi|$ impossible. We conclude that the average overspatial translations is already performed in (2.9). Since one of the two available length scales $\rho$ and $\beta$ parametrizing the caloron or the anticaloron is integrated over in (2.9), the only scale responsible for a nontrivial $\tau$ dependence of $\phi^{a} /|\phi|$ is $\beta$.

What about the contribution of calorons with a topological charge modulus larger than unity? Let us consider the charge-two case. Here we have three moduli of dimension length which should enter the average defining the differential operator $\Phi$ : two scale parameters and a core separation. The reader may easily convince himself that by the absence of an explicit temperature dependence it is impossible to define the associated dimensionless quantity in terms of spatial and moduli averages over $n$-point functions involving these configurations. The situation is even worse for calorons of topological charge larger than two. We conclude that only calorons of topological charge \pm 1 contribute to the definition of the operator $\Phi$ in (2.14) by means of (2.9). 


\section{Computation of Two-Point Correlation}

Before we perform the actual calculation let us stress some simplifying properties of the solutions $A_{\mu}^{(C, A)}$ in (2.2).

The path-ordering prescription for the Wilson lines $\{(\tau, 0),(\tau, \mathbf{x})\}$ and $\{(\tau, 0),(\tau, \mathbf{x})\}^{\dagger}$ in (2.10) can actually be omitted. To see this, we first consider the quantity $P^{(C, A)}(\tau, r s t)$ defined as

$$
P^{(C, A)}(\tau, r s \mathbf{t}) \equiv A_{i}^{(C, A)}(\tau, s r \mathbf{t}) t_{i}
$$

where $0 \leq r \leq \infty, 0 \leq s \leq 1,(i=1,2,3)$. The vector $\mathbf{t}$ denotes the unit line tangential along the straight line connecting the points $(\tau, 0)$ and $(\tau, \mathbf{x}) \equiv(\tau, r \mathbf{t})$. We have

$$
\{(\tau, 0),(\tau, r \mathbf{t})\}^{(C, A)}=p \exp \left[\operatorname{ir} \int_{0}^{1} d s P^{(C, A)}(\tau, s r t)\right]
$$

where

$$
P^{(C, A)}(\tau, s r t)=\mp \frac{1}{2} \mathfrak{t} \cdot \lambda \partial_{4} \ln \Pi(\tau, s r)
$$

Thus the path-ordering symbol can, indeed, be omitted in (3.2). The field strength $F_{a \mu \nu}^{C}$ on the caloron solution in (2.2) is

$$
\begin{aligned}
F_{a \mu \nu}^{C}= & \bar{\eta}_{a \mu \nu} \frac{\left(\partial_{\kappa} \Pi\right)\left(\partial_{\kappa} \Pi\right)}{\Pi^{2}}+\bar{\eta}_{a \mu \kappa} \frac{\Pi\left(\partial_{\nu} \partial_{\kappa} \Pi\right)-2\left(\partial_{\kappa} \Pi\right)\left(\partial_{v} \Pi\right)}{\Pi^{2}} \\
& -\bar{\eta}_{a v \kappa} \frac{\Pi\left(\partial_{\mu} \partial_{\kappa} \Pi\right)-2\left(\partial_{\kappa} \Pi\right)\left(\partial_{\mu} \Pi\right)}{\Pi^{2}}
\end{aligned}
$$

where $\Pi$ is defined in (2.4). For the anticaloron one replaces $\bar{\eta}$ by $\eta$ in (3.4). Using (2.9), (3.2), and (3.4), we obtain the following expression for the contribution $\left.\left(\phi^{a} /|\phi|\right)\right|_{C}$ arising from calorons:

$$
\begin{aligned}
\left.\frac{\phi^{a}}{|\phi|}\right|_{C} \sim & i \int d \rho \int d^{3} x \frac{x^{a}}{r}\left[\frac{\left(\partial_{4} \Pi(\tau, 0)\right)^{2}}{\Pi^{2}(\tau, 0)}-\frac{2}{3} \frac{\partial_{4}^{2} \Pi(\tau, 0)}{\Pi(\tau, 0)}\right] \\
\times & \left\{4 \cos (2 g(\tau, r))\left[\frac{\partial_{r} \partial_{4} \Pi(\tau, r)}{\Pi(\tau, r)}-2 \frac{\left(\partial_{r} \Pi(\tau, r)\right)\left(\partial_{4} \Pi(\tau, r)\right)}{\Pi^{2}(\tau, r)}\right]\right. \\
& \left.+\sin (2 g(\tau, r))\left[4 \frac{\left(\partial_{4} \Pi(\tau, r)\right)^{2}-\left(\partial_{r} \Pi(\tau, r)\right)^{2}}{\Pi^{2}(\tau, r)}+2 \frac{\partial_{r}^{2} \Pi(\tau, r)-\partial_{4}^{2} \Pi(\tau, r)}{\Pi(\tau, r)}\right]\right\},
\end{aligned}
$$


where

$$
\begin{gathered}
g(\tau, r) \equiv \int_{0}^{1} d s \frac{r}{2} \partial_{4} \ln \Pi(\tau, s r), \\
\frac{\left(\partial_{4} \Pi(\tau, 0)\right)^{2}}{\Pi^{2}(\tau, 0)}-\frac{2}{3} \frac{\partial_{4}^{2} \Pi(\tau, 0)}{\Pi(\tau, 0)}=-\frac{16}{3} \pi^{4} \frac{\rho^{2}}{\beta^{2}} \frac{\pi^{2} \rho^{2}+\beta^{2}(2+\cos (2 \pi \tau / \beta))}{\left[2 \pi^{2} \rho^{2}+\beta^{2}(1-\cos (2 \pi \tau / \beta))\right]^{2}} .
\end{gathered}
$$

The dependences on $\rho$ and $\beta$ are suppressed in the integrands of (3.5) and (3.6). It is worth mentioning that the integrand in (3.6) is proportional to $\delta(s)$ for $r \gg \beta$. A useful set of identities is

$$
\begin{aligned}
F_{\mu \nu}^{C}(\tau, \mathbf{x}) & =F_{\mu \nu}^{A}(\tau,-\mathbf{x}) \\
\{(\tau, 0),(\tau, \mathbf{x})\}^{C} & =\left(\{(\tau, \mathbf{x}),(\tau, 0)\}^{C}\right)^{\dagger}=\{(\tau, 0),(\tau,-\mathbf{x})\}^{A} \\
& =\left(\left\{(\tau,-\mathbf{x}),(\tau, 0\}^{A}\right)^{\dagger}\right.
\end{aligned}
$$

Equation (3.8) states that the integrand for $\left.\left(\phi^{a} /|\phi|\right)\right|_{A}$ can be obtained by a parity transformation $\mathbf{x} \rightarrow-\mathbf{x}$ of the integrand for $\left.\left(\phi^{a} /|\phi|\right)\right|_{C}$. Since the latter changes its sign, see (3.5); one naively would conclude that

$$
\frac{\phi^{a}}{|\phi|}=\left.\frac{\phi^{a}}{|\phi|}\right|_{C}+\left.\frac{\phi^{a}}{|\phi|}\right|_{A}=0
$$

This, however, would only be the case if no ambiguity in evaluating the integral in both cases existed. But such ambiguities do occur! First, the $\tau$ dependence of the anticaloron's contribution may be shifted as compared to that of the caloron. Second, the color orientation of caloron and anticaloron contributions may be different. Third, the normalization of the two contributions may be different. To see this, we need to investigate the convergence properties of the radial integration in (3.5). It is easily checked that all terms give rise to a converging $r$ integration except for the following one:

$$
2 \frac{x^{a}}{r} \sin (2 g(\tau, r)) \frac{\partial_{r}^{2} \Pi(\tau, r)}{\Pi(\tau, r)}
$$

Namely, for $r>R \gg \beta$ (3.10) goes over in

$$
4 t^{a} \frac{\pi \rho^{2} \sin (2 g(\tau, r))}{\beta r^{3}} .
$$

Thus the $r$-integral of the term in (3.10) is logarithmically divergent in the infrared ${ }^{13}$ :

$$
4 t^{a} \frac{\pi \rho^{2}}{\beta} \int_{R}^{\infty} \frac{d r}{r} \sin (2 g(\tau, r))
$$


Recall that $g(\tau, r)$ behaves like a constant in $r$ for $r>R$. The angular integration, on the other hand, would yield zero if the radial integration was regular. Thus a logarithmic divergence can be cancelled by the vanishing angular integral to yield some finite and real but undetermined normalization of the emerging $\tau$ dependence. To investigate this, both angular and radial integration need to regularized.

We may regularize the $r$ integral in (3.12) by prescribing

$$
\int_{R}^{\infty} \frac{d r}{r} \longrightarrow \beta^{\epsilon} \int_{R}^{\infty} \frac{d r}{r^{1+\epsilon}}
$$

with $\epsilon>0$. We have

$$
\begin{aligned}
\beta^{\epsilon} \int_{R}^{\infty} \frac{d r}{r^{1+\epsilon}} & =\beta^{\epsilon} \int_{0}^{\infty} \frac{d r}{(r+R)^{1+\epsilon}} \\
& =\frac{1}{\epsilon}-\log \left(\frac{R}{\beta}\right)+\frac{1}{2} \epsilon \log ^{2}\left(\frac{R}{\beta}\right)+\cdots
\end{aligned}
$$

Away from the pole at $\epsilon=0$ this is regular. For $\epsilon<0$ (3.14) can be regarded as a legitimate analytical continuation. An ambiguity inherent in (3.14) relates to how one circumvents the pole in the smeared expression

$$
\begin{gathered}
\frac{1}{2 \eta} \int_{-\eta}^{\eta} d \epsilon\left(\frac{1}{\epsilon \pm i 0}-\log \left(\frac{R}{\beta}\right)+\frac{1}{2} \epsilon \log ^{2}\left(\frac{R}{\beta}\right)+\cdots\right) \\
=\mp \frac{\pi i}{2 \eta}-\log \left(\frac{R}{\beta}\right)+\cdots, \quad(\eta>0, \eta \ll 1) .
\end{gathered}
$$

Concerning the regularization of the angular integration we may introduce defect (or surplus) angles $2 \eta^{\prime}$ in the azimuthal integration as

$$
\int_{0}^{\pi} d \omega \sin \omega \int_{0}^{2 \pi} d \theta \longrightarrow \int_{0}^{\pi} d \omega \sin \omega \int_{\alpha_{C} \pm \eta^{\prime}}^{\alpha_{C}+2 \pi \mp \eta^{\prime}} d \theta
$$

see Figure 3. In (3.16) $\alpha_{C}$ is a constant angle with $0 \leq \alpha_{C} \leq 2 \pi$ and $0<\eta^{\prime} \ll 1$. Obviously, this regularization singles out the $x_{1} x_{2}$ plane. As we will show below, the choice of regularization plane translates into a global gauge choice for the $\tau$ dependence of $\phi^{\prime}$ s phase and thus is physically irrelevant: the apparent breaking of rotational symmetry by the angular regularization translates into a gauge choice.

The value of $\alpha_{C}$ is determined by an (physically irrelevant) initial condition. We have

$$
\int_{0}^{\pi} d \omega \sin \omega \int_{\alpha_{C} \pm \eta^{\prime}}^{\alpha_{C}+2 \pi \mp \eta^{\prime}} d \theta t^{a} \sim \mp \pi \eta^{\prime}\left(\delta_{a 1} \cos \alpha_{C}+\delta_{a 2} \sin \alpha_{C}\right) .
$$




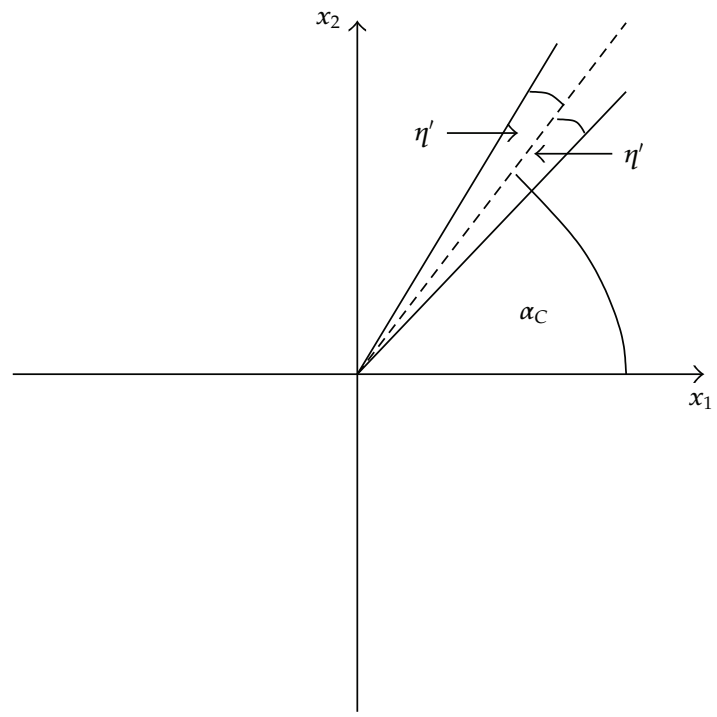

Figure 3: The axis for the integration over $\theta$.

To see what is going on we may fix, for the time being, the ratio $\eta^{\prime} / \eta$ for the normalization of the caloron contribution to a finite and positive but otherwise arbitrary constant $\Xi$ when sending $\eta$ and $\eta^{\prime}$ to zero in the end of the calculation:

$$
\lim _{\eta, \eta^{\prime} \rightarrow 0} \frac{\eta^{\prime}}{\eta}=\Xi
$$

Combining (3.12), (3.15), (3.17), and (3.7), expression (3.5) reads:

$$
\begin{aligned}
\left.\frac{\phi^{a}}{|\phi|}\right|_{C} \sim & \pm \frac{32}{3} \frac{\pi^{7}}{\beta^{3}} \Xi\left(\delta_{a 1} \cos \alpha_{C}+\delta_{a 2} \sin \alpha_{C}\right) \int d \rho\left[\lim _{r \rightarrow \infty} \sin (2 g(\tau, r))\right] \\
& \times \rho^{4} \frac{\pi^{2} \rho^{2}+\beta^{2}(2+\cos (2 \pi \tau / \beta))}{\left[2 \pi^{2} \rho^{2}+\beta^{2}(1-\cos (2 \pi \tau / \beta))\right]^{2}} \\
\equiv & \pm \Xi\left(\delta_{a 1} \cos \alpha_{C}+\delta_{a 2} \sin \alpha_{C}\right) A\left(\frac{2 \pi \tau}{\beta}\right),
\end{aligned}
$$

where $\mathcal{A}$ is a dimensionless function of its dimensionless argument. The sign ambiguity in (3.19) arises from the ambiguity associated with the way how one circumvents the pole in (3.15) and whether one introduces a surplus or a defect angle in (3.16). Furthermore, there is an ambiguity associated with a constant shift $\tau \rightarrow \tau+\tau_{C}\left(0 \leq \tau_{C} \leq \beta\right)$ in (3.19).

For the anticaloron contribution we may, for the time being, fix the ratio $\eta^{\prime} / \eta$ to another finite and positive constant $\Xi^{\prime}$. In analogy to the caloron case, there is the ambiguity related to a shift $\tau \rightarrow \tau+\tau_{A}\left(0 \leq \tau_{A} \leq \beta\right)$ in the anticaloron contribution. Moreover, we may without 
restriction of generality (global gauge choice) use an axis for the angular regularization which also lies in the $x_{1} x_{2}$ plane, but with a different angle $\alpha_{A}$. Then we have

$$
\begin{aligned}
\frac{\phi^{a}}{|\phi|}= & \left.\frac{\phi^{a}}{|\phi|}\right|_{C}+\left.\frac{\phi^{a}}{|\phi|}\right|_{A} \\
= & \pm \Xi\left(\delta_{a 1} \cos \alpha_{C}+\delta_{a 2} \sin \alpha_{C}\right) \mathcal{A}\left(\frac{2 \pi\left(\tau+\tau_{C}\right)}{\beta}\right) \\
& \pm \Xi^{\prime}\left(\delta_{a 1} \cos \alpha_{A}+\delta_{a 2} \sin \alpha_{A}\right) \mathcal{A}\left(\frac{2 \pi\left(\tau+\tau_{A}\right)}{\beta}\right)
\end{aligned}
$$

$\neq 0$,

where the choices of signs in either contribution are independent. Equation (3.20) is the basis for fixing the operator $\Phi$ in (2.14).

To evaluate the function $\mathcal{A}(2 \pi \tau / \beta)$ in (3.19) numerically, we introduce the same cutoff for the $\rho$ integration in the caloron and anticaloron case as follows:

$$
\int d \rho \longrightarrow \int_{0}^{\zeta \beta} d \rho, \quad(\zeta>0)
$$

This introduces an additional dependence of $\mathcal{A}$ on $\zeta$. In Figure 4 the $\tau$ dependence of $\mathscr{A}$ for various values of $\zeta$ is depicted. It can be seen that

$$
\mathcal{A}\left(\frac{2 \pi}{\beta} \tau, \zeta \rightarrow \infty\right) \longrightarrow 272 \zeta^{3} \sin \left(\frac{2 \pi}{\beta} \tau\right)
$$

Therefore we have

$$
\begin{aligned}
\frac{\phi^{a}}{|\phi|} \sim 272 \zeta^{3}( & \pm \Xi\left(\delta_{a 1} \cos \alpha_{C}+\delta_{a 2} \sin \alpha_{C}\right) \sin \left(\frac{2 \pi}{\beta}\left(\tau+\tau_{C}\right)\right) \\
& \left. \pm \Xi^{\prime}\left(\delta_{a 1} \cos \alpha_{A}+\delta_{a 2} \sin \alpha_{A}\right) \sin \left(\frac{2 \pi}{\beta}\left(\tau+\tau_{A}\right)\right)\right) \\
\equiv \widehat{\phi}^{a} . &
\end{aligned}
$$

The numbers $\zeta^{3} \Xi, \zeta^{3} \Xi^{\prime}, \tau_{C} / \beta$, and $\tau_{A} / \beta$ in (3.23) are undetermined. For each color orientation (corresponding to a given angular regularization) there are two independent parameters, a normalization and a phase shift. The principal impossibility to fix the normalizations reflects the fact that on the classical level the theory is invariant under spatial dilatations. To give a meaning to these number, a mass scale needs to be generated dynamically. This, however, can only happen due to dimensional transmutation, which is known to be an effect induced by trivial-topology fluctuations [8-11]. The result in (3.23) is highly nontrivial since it is obtained only after an integration over the entire admissible part of the moduli spaces of (anti)calorons is performed. 


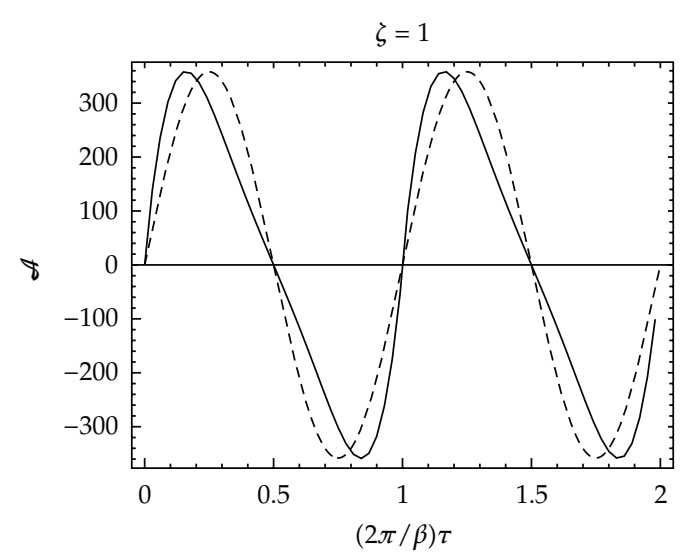

(a)

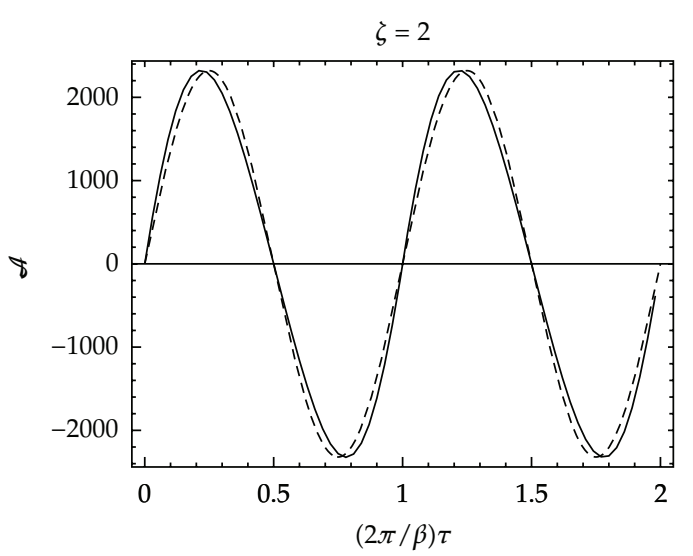

(b)

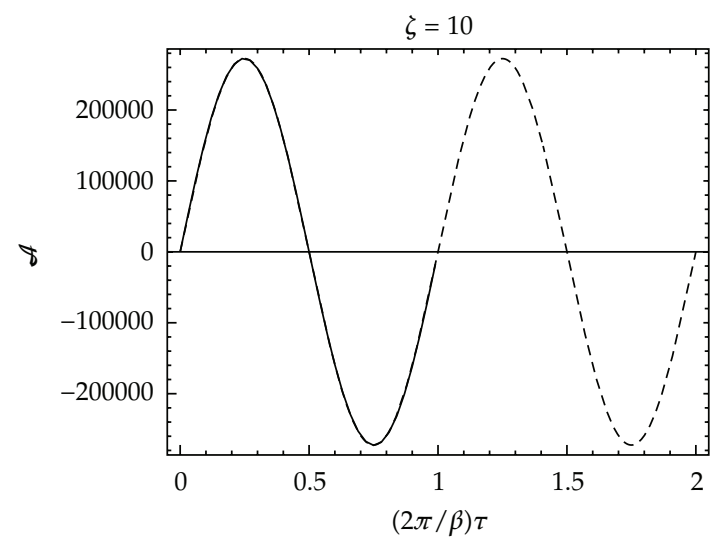

(c)

Figure 4: $\mathcal{A}$ as a function of $(2 \pi / \beta \tau)$ for $\zeta=1,2,10$. For each case the dashed line is a plot of $\max \mathscr{A} \times$ $\sin ((2 \pi / \beta) \tau)$. We have fitted the asymptotic dependence on $\zeta$ of the amplitude of $\mathcal{A}$ as $\mathcal{A}((2 \pi / \beta) \tau=$ $\pi / 2, \zeta)=272 \zeta^{3},(\zeta>10)$. The fit is stable under variations of the fitting interval. For the case $\zeta=10$ the difference between the two curves cannot be resolved anymore.

Let us now discuss the physical content of (3.23). For fixed values of the parameters $\zeta^{3} \Xi, \zeta^{3} \Xi^{\prime}, \tau_{C} / \beta$, and $\tau_{A} / \beta$ the right-hand side of (3.23) resembles a fixed elliptic polarization in the $x_{1} x_{2}$ plane of adjoint color space. For a given polarization plane the two independent numbers (normalization and phase-shift) of each oscillation axis parametrize the solution space (in total four undetermined parameters) of a second-order linear differential equation

$$
\Phi \widehat{\phi}=0
$$

From (3.23) we observe that the operator $\Phi$ is

$$
\Phi=\partial_{\tau}^{2}+\left(\frac{2 \pi}{\beta}\right)^{2}
$$


Since for a given polarization plane there is a one-to-one map from the solution space of (3.24) to the parameter space associated with the ambiguities in the definition (2.9) we conclude that the operator $\Phi$ is uniquely determined by (2.9).

What we need to assure the validity of (2.7) is a BPS saturation ${ }^{14}$ of the solution to (3.24). Thus we need to find first-order equations whose solutions solve the second-order equation (3.24). The relevant two first-order equations are

$$
\partial_{\tau} \widehat{\phi}= \pm \frac{2 \pi i}{\beta} \lambda_{3} \widehat{\phi}
$$

where we have defined $\phi=|\phi| \widehat{\phi}(\tau)$. Obviously, the right-hand sides of (3.26) are subject to a global gauge ambiguity associated with the choice of plane for angular regularization, any normalized generator other than $\lambda_{3}$ could have appeared, see Figure 2. Now the solution to either of the two equations (3.26) also solves (3.24),

$$
\begin{aligned}
\partial_{\tau}^{2} \widehat{\phi} & = \pm \frac{2 \pi i}{\beta} \lambda_{3} \partial_{\tau} \widehat{\phi} \\
& =\frac{2 \pi i}{\beta} \lambda_{3} \frac{2 \pi \mathrm{i}}{\beta} \lambda_{3} \widehat{\phi} \\
& =-\left(\frac{2 \pi}{\beta}\right)^{2} \widehat{\phi} .
\end{aligned}
$$

Traceless, hermitian solutions to (3.26) are given as

$$
\widehat{\phi}=C \lambda_{1} \exp \left(\mp \frac{2 \pi i}{\beta} \lambda_{3}\left(\tau-\tau_{0}\right)\right)
$$

where $C$ and $\tau_{0}$ denote real integration constants which both are undetermined. Notice that the requirement of BPS saturation has reduced the number of undetermined parameters from four to two: an elliptic polarization in the $x_{1} x_{2}$ plane is cast into a circular polarization. Thus the field $\phi$ winds along an $S^{1}$ on the group manifold $S^{3}$ of $S U(2)$. Both winding senses appear but cannot be distinguished physically $[1,2]$.

\section{How to Obtain $\phi$ 's Modulus}

Here we show how the information about $\phi$ 's phase in (3.28) can be used to infer its modulus. Let us assume that a scale $\Lambda$ is externally given which characterizes this modulus at a given temperature $T$. Together, $\Lambda$ and $T$ determine what the minimal physical volume $|\phi|^{-3}$ is for which the spatial average over the caloron-anticaloron system saturates the infinite-volume average appearing in (2.9).

We have

$$
\phi=\phi\left(\beta, \Lambda, \frac{\tau}{\beta}\right) .
$$


In order to reproduce the phase in (3.28) a linear dependence on $\phi$ must appear on the righthand side of the BPS equation (2.8). Furthermore, this right-hand side ought not depend on $\beta$ explicitly and must be analytic in $\phi^{15}$. The two following possibilities exist:

$$
\partial_{\tau} \phi= \pm i \Lambda \lambda_{3} \phi
$$

or

$$
\partial_{\tau} \phi= \pm i \Lambda^{3} \lambda_{3} \phi^{-1}
$$

where $\phi^{-1} \equiv \phi /|\phi|^{2}$. Recall that

$$
\phi^{-1}=\phi_{0}^{-1} \sum_{n=0}^{\infty}(-1)^{n} \phi_{0}^{-n}\left(\phi-\phi_{0}\right)^{n}
$$

has a finite radius of convergence. According to (3.28) we may write

$$
\phi=|\phi|(\beta, \Lambda) \times \lambda_{1} \exp \left(\mp \frac{2 \pi i}{\beta} \lambda_{3}\left(\tau-\tau_{0}\right)\right) .
$$

Substituting (4.5) into (4.2) yields

$$
\Lambda=\frac{2 \pi}{\beta}
$$

which is unacceptable since $\Lambda$ is a constant scale. For the possibility in (4.3) we obtain

$$
|\phi|(\beta, \Lambda)=\sqrt{\frac{\beta \Lambda^{3}}{2 \pi}}=\sqrt{\frac{\Lambda^{3}}{2 \pi T}}
$$

when substituting (4.5) into (4.3). This is acceptable and indicates that at $T \gg \Lambda \phi^{\prime}$ s modulus is small. The right-hand side of (4.3) defines the "square-root" $V^{(1 / 2)}$ of a potential $V(|\phi|) \equiv \operatorname{tr}\left(V^{(1 / 2)}\right)^{\dagger} V^{(1 / 2)}=\Lambda^{6} \operatorname{tr} \phi^{-2}$, and the equation of motion (4.3) can be derived from the following action:

$$
S_{\phi}=\operatorname{tr} \int_{0}^{\beta} d \tau \int d^{3} x\left(\partial_{\tau} \phi \partial_{\tau} \phi+\Lambda^{6} \phi^{-2}\right)
$$

Notice that a shift $V \rightarrow V+$ const is forbidden in (4.8) since the relevant equation of motion is the first-order equation (4.3).

After the spatial average is performed the action $S_{\phi}$ is extended by including topologically trivial configurations $a_{\mu}$ in a minimal fashion: $\partial_{\tau} \phi \rightarrow \partial_{\mu} \phi+i e\left[\phi, a_{\mu}\right] \equiv D_{\mu} \phi$ 
and an added kinetic term. Here $e$ denotes the effective gauge coupling. Thus the effective Yang-Mills action $S$ is written as

$$
S=\operatorname{tr} \int_{0}^{\beta} d \tau \int d^{3} x\left(\frac{1}{2} G_{\mu \nu} G_{\mu \nu}+D_{\mu} \phi D_{\mu} \phi+\Lambda^{6} \phi^{-2}\right)
$$

where $G_{\mu \nu}=G_{\mu \nu}^{a} \lambda^{a} / 2$ and $G_{\mu \nu}^{a}=\partial_{\mu} a_{\nu}^{a}-\partial_{\nu} a_{\mu}^{a}-e \epsilon^{a b c} a_{\mu}^{b} a_{\nu}^{c}$.

In (4.2) and (4.3) the existence of the mass scale $\Lambda$ (the Yang-Mills scale) was assumed. One attributes the generation of a mass scale to the topologically trivial sector which, however, was assumed to be switched off so far. How can a contradiction be avoided? The answer to this question is that the scale $\Lambda$ remains hidden as long as topologically trivial fluctuations are switched off; see (2.7). Only after switching on interactions between trivialholonomy calorons within the ground state can $\Lambda$ be seen $[1,2]$. Let us repeat the derivation of this result: in $[1,2]$ we have shown that the mass squared of $\phi$-field fluctuations, $\partial_{|\phi|}^{2} V(|\phi|)$, is much larger than the square of the compositeness scale $|\phi|$. Moreover $\partial_{|\phi|}^{2} V(|\phi|)$ is much larger than $T^{2}$ for all temperatures $T \geq T_{c, E}$ where $T_{c, E}$ denotes the critical temperature for the electric-magnetic transition. Thus $\phi$ is quantum mechanically and statistically inert: it provides a (nonbackreacting and undeformable) source for the following equation of motion:

$$
\Phi_{\mu} G_{\mu \nu}=2 i e\left[\phi, \Phi_{\nu} \phi\right]
$$

which follows from the action in (4.9). A pure-gauge solution to (4.10), describing the ground state together with $\phi$, is

$$
a_{\mu}^{b g}=\frac{\pi}{e} T \delta_{\mu 4} \lambda_{3}
$$

As a consequence of (4.11) we have $\Phi_{\mu} \phi \equiv 0$, and thus a ground-state pressure $P^{\text {g.s. }}=-4 \pi \Lambda^{3} T$ and a ground-state energy-density $\rho^{\text {g.s. }}=4 \pi \Lambda^{3} T$ are generated in the electric phase: The so far hidden scale $\Lambda$ becomes visible by averaged-over caloron-anticaloron interactions encoded in the pure-gauge configuration $a_{\mu}^{b g}$.

\section{Summary and Outlook}

Let us summarize our results. We have derived the phase and the modulus of a statistically and quantum mechanically inert adjoint and spatially homogeneous scalar field $\phi$ for an $\mathrm{SU}(2)$ Yang-Mills theory being in its electric phase. This field and a pure-gauge configuration together suggest the concept of a thermal ground state since they generate temperaturedependent pressure and energy density with an equation of state corresponding to a cosmological constant. The existence of $\phi$ originates from the spatial correlations inherent in BPS saturated, trivial-holonomy solutions to the classical Yang-Mills equations at finite temperature: the Harrington-Shepard solutions of topological charge modulus one. To derive $\phi^{\prime} \mathrm{s}$ phase these field configurations are, in a first step, treated as noninteracting when performing the functional average over the admissible parts of their moduli spaces. We have shown why adjoint scalar fields arising from configurations of higher topological charge do not exist. 
The BPS saturated and classical field $\phi$ possesses nontrivial $S^{1}$ winding on the group manifold $S^{3}$. The associated trajectory on $S^{3}$ becomes circular and thus a pure phase only after the integration over the entire admissible parts of the moduli spaces is carried out. Together with a pure-gauge configuration the adjoint scalar field $\phi$ generates a linear temperature dependence of the ground-state pressure and the ground-state energy density where the pure-gauge configuration solves the Yang-Mills equations in the background $\phi$ and, after the spatial average, describes the interactions between trivial-holonomy calorons. The puregauge configuration also makes explicit that the electric phase is deconfining [1,2]. Since trivial-topology fluctuations may acquire quasiparticle masses on tree level by the adjoint Higgs mechanism [1,2], the presence of $\phi$ resolves the infrared problem inherent in a perturbative loop expansion of thermodynamical quantities [27]. Since there are kinematical constraints for the maximal hardness of topologically trivial quantum fluctuations, no renormalization procedure for the treatment of ultraviolet divergences is needed in the loop expansion of thermodynamical quantities [27] performed in the effective theory. These kinematical constraints arise from $\phi^{\prime}$ s compositeness emerging at distances $\sim|\phi|^{-1}$. The usual assertion that the effects of the topologically nontrivial sector are extremely suppressed at high temperature-they turn out to be power suppressed in $T$-is shown to be correct by taking this sector into account. The theory, indeed, has a Stefan-Boltzmann limit which is very quickly approached. It turns out to be incorrect, however, to neglect the topologically nontrivial sector from the start: assuming $T \gg \Lambda$ to justify the omission of the topologically nontrivial sector before performing a (perturbative) loop expansion of thermodynamical quantities does not capture the thermodynamics of an SU(2) Yang-Mills theory and leads to the known problems in the infrared sector [28].

\section{Acknowledgments}

The authors would like to thank Nucu Stamatescu for his continuing interest in our work, and Janos Polonyi and Dirk Rischke for stressing the necessity of this paper. Useful conversations with H. Gies and J. Pawlowski are gratefully acknowledged.

\section{Endnotes}

1. By "condensation" we mean the effects of long-range spatial correlations in the classical, BPS-saturated, trivial-holonomy configurations in singular gauge, see (2.9).

2. In unitary gauge off-Cartan fluctuations acquire a temperature-dependent mass when propagating through and thereby interacting with the "medium" of caloron fluctuations.

3. The reader may convince himself of this fact by computing the energy-momentum tensor on a BPS monopole.

4. Gauge-field excitations are free at large temperatures [27] and contribute to the total pressure and the total energy density like $\sim T^{4}$. The small residual interactions, which peak close to a 2nd-order transition to the magnetic phase at $T_{c, E}$, are likely to explain the large-angle anomalies seen in some CMB power spectra [27, 29]. When cooling the system, monopoles and antimonopoles, which were generated by dissociating calorons (large holonomy), start to overlap at a temperature $T_{o}$ slightly higher than $T_{c, E}$ because they are moving towards one another under the influence of an overall negative pressure. 
The latter is generated by the dominating pressure component generated by smallholonomy calorons whose monopole constituents attract and eventually annihilate at a given point in space but get recreated elseswhere. Naively seen, negative pressure corresponds to an instability of the system causing it to collapse. We usually imagine a contracting system in terms of a decrease of the mean interparticle distance while tacitly assuming the particles to be pointlike. Despite an overall negative pressure a total collapse in the above sense does not occur in an SU(2) Yang-Mills theory. This can be understood as follows: the mass of an isolated, screened monopole is $m \sim 2 \pi^{2} T / e$, and the effective gauge coupling $e$ is constant if monopoles do not overlap, that is, for $T>T_{o}$ (magnetic charge conservation $[1,2]$ ). At $T_{o}$ the magnetic charge contained in a given spatial volume no longer remains constant in time because of the increasing mobility of strongly screened monopoles. Thus formerly separated monopoles can annihilate and but also get recreated. Therefore the notion of a local collapse ceases to be applicable since the associated particles cease to exist if they are close to one another. If the rate of annihilation equals the rate of recreation of monopole-antimonopole pairs then we are witnessing an equilibium situation characterized by a temperature despite a negative overall pressure.

5. Configurations with higher topological charge and trivial holonomy have been constructed, see for example [30,31]. A priori they should contribute to the ground-state thermodynamics of the theory in terms of additional adjoint scalar fields. The nonexistence of these Higgs fields is assured by their larger number of dimensionful moduli-for the charge-two case we have two instanton radii and the core separation-which does not allow for the nonlocal definition of a macroscopic, dimensionless phase; see (2.9) and the discussion following it.

6. Because of periodicity, $\tau_{z}$ needs to be restricted as $0 \leq \tau_{z} \leq \beta$.

7. A spatial average over zero energy momentum yields zero.

8. The fact that an ordinary and not a covariant derivative appears in (2.8) is, of course, tied to our specific gauge choice. If we were to leave the (singular) gauge for the (anti)instanton, in which the solutions of (2.2) are constructed, by a time-dependent gauge rotation $\bar{\Omega}(\tau)$ then a pure-gauge configuration $A_{\mu}^{\text {p.g. }}(\tau)=i \delta_{\mu 4} \bar{\Omega}^{\dagger} \partial_{\tau} \bar{\Omega}$ would appear in a covariant derivative on the left-hand side of (2.8).

9. A local definition of $\phi^{\prime}$ s phase always yields zero due to the (anti)self-duality of the (anti)caloron configuration.

10. Curved integration contours introduce scales which have no physical counterpart on the classical level. Furthermore, shifting the spatial part of the argument $(\tau, 0) \rightarrow(\tau, \mathbf{z})$ in (2.9) introduces a parameter $|\mathbf{z}|$ of dimension inverse mass. There is no physical reason for a finite value of $|\mathbf{z}|$ to exist on the classical level. Thus we conclude that $\mathbf{z}=0$.

11. A shift, $\tau \rightarrow \tau+\tau_{z}\left(0 \leq \tau_{z} \leq \beta\right)$, is always possible and not fixed by a physical boundary condition on the classical level. As we will see below, the same holds true for the normalization of the right-hand side of (2.9). Therefore, for each color direction these two ambiguities parametrize the solution space of the second-order linear operator $\Phi$.

12. The "naked" gauge charge in (2.9) is needed for a coupling of the trivial topology sector to the ground-state after spatial coarse-graining generating (i) quasiparticle masses and (ii) finite values of the ground-state energy density and the ground-state pressure $[1,2]$. 
13. The integral converges for $r \rightarrow 0$.

14. The modulus $|\phi|$ does not depend on $\tau$ in thermal equilibrium and thus can be cancelled out.

15. The former requirement derives from the fact that $\phi$ and its potential $V$ are obtained by functionally integrating over a noninteracting caloron-anticaloron system. The associated part of the partition function does not exhibit an explicit $\beta$ dependence since the action $S$ is $\beta$ independent on the caloron and anticaloron moduli spaces. Thus a $\beta$ dependence of $V$ or $V^{(1 / 2)}$ can only be generated via the periodicity of $\phi$ itself. The latter requirement derives from the demand that the thermodynamics at temperature $T+\delta T$ to any given accuracy must be derivable from the thermodynamics at temperature $T$ for $\delta T$ sufficiently small provided no phase transition occurs at $T$. This is accomplished by a Taylor expansion of the right-hand side of the BPS equation (finite radius of convergence) which, in turn, is the starting point for a perturbative treatment with expansion parameter $\delta T / T$.

\section{References}

[1] R. Hofmann, "Nonperturbative approachto Yang-Mills thermodynamics," International Journal of Modern Physics A, vol. 20, no. 18, pp. 4123-4216, 2005.

[2] R. Hofmann, "Erratum: nonperturbative approach to Yang-Mills thermodynamics (International Journal of Modern Physics A (2005) 20, 18, (4123-4216))," International Journal of Modern Physics A, vol. 21, no. 31, pp. 6515-6523, 2006.

[3] B. J. Harrington and H. K. Shepard, "Periodic euclidean solutions and the finite-temperature YangMills gas," Physical Review D, vol. 17, no. 8, pp. 2122-2125, 1978.

[4] G. 't Hooft and M. Veltman, "Regularization and renormalization of gauge fields," Nuclear Physics B, vol. 44, no. 1, pp. 189-213, 1972.

[5] G. 't Hooft, "Renormalization of massless Yang-Mills fields," Nuclear Physics B, vol. 33, no. 1, pp. 173-199, 1971.

[6] G. 't Hooft, "An algorithm for the poles at dimension four in the dimensional regularization procedure," Nuclear Physics B, vol. 62, pp. 444-460, 1973.

[7] G. 't Hooft and M. J. G. Veltman, "Combinatorics of gauge fields," Nuclear Physics B, vol. 50, no. 1, pp. 318-353, 1972.

[8] D. J. Gross and F. Wilczek, “Asymptoticallyfree gauge theories. I," Physical Review D, vol. 8, no. 10, pp. 3633-3652, 1973.

[9] D. J. Gross and F. Wilczek, "Ultraviolet behavior of non-abelian gauge theories," Physical Review Letters, vol. 30, no. 26, pp. 1343-1346, 1973.

[10] H. D. Politzer, "Reliable perturbative results for strong interactions?" Physical Review Letters, vol. 30, no. 26, pp. 1346-1349, 1973.

[11] H. D. Politzer, "Asymptotic freedom: an approach to strong interactions," Physics Reports, vol. 14, no. 4, pp. 129-180, 1974.

[12] W. Nahm, "A simple formalism forthe BPS monopole," Physics Letters B, vol. 90, no. 4, pp. 413-414, 1980.

[13] W. Nahm, "Self-dual monopoles and calorons," in Lecture Notes in Physics, G. Denaro, Ed., vol. 201, pp. 189-200, 1984.

[14] K. Lee and C. Lu, “Su(2) calorons and magnetic monopoles," Physical Review D, vol. 58, pp. 0250111-025011-7, 1998.

[15] T. C. Kraan and P. van Baal, "Periodic instantons with non-trivial holonomy," Nuclear Physics B, vol. 533, no. 1-3, pp. 627-659, 1998.

[16] T. C. Kraan and P. van Baal, "Monopole constituents inside SU(n) calorons," Physics Letters B, vol. 435, no. 3-4, pp. 389-395, 1998.

[17] R. C. Brower, D. Chen, J. Negele, K. Orginos, and C.-I. Tan, "Magnetic monopole content of hot instantons," Nuclear Physics B-Proceedings Supplements, vol. 73, no. 1-3, pp. 557-559, 1999. 
[18] D. Diakonov, N. Gromov, V. Petrov, and S. Slizovskiy, "Quantum weights of dyons and of instantons with nontrivial holonomy," Physical Review D, vol. 70, no. 3, Article ID 036003, 2004.

[19] C. P. K. Altes, "Spatial 't Hooft loop,hot QCD and Z_N domain walls," Physics Letters B, vol. 469, no. 1-4, pp. 205-212, 1999.

[20] C. P. K. Altes, "Quasi-particles and strong first order transition in hot QCD," Acta Physica Polonica B, vol. 34, no. 12, pp. 5825-5845, 2003.

[21] P. Giovannangeli and C. P. K. Altes, "'T Hooft and Wilson loop ratios in the QCD plasma," Nuclear Physics B, vol. 608, no. 1-2, pp. 203-234, 2001.

[22] C. P. K. Altes, "Spatial 't Hooft loop, hot QCD and Z_N domain walls," Physics Letters B, vol. 469, no. $1-4$, pp. 205-212, 1999.

[23] C. H. Hoelbing, C. Rebbi, and V. A. Rubakov, "Free energy of an SU(2) monopole-antimonopole pair," Physical Review D, vol. 63, no. 3, Article ID 034506, 8 pages, 2001.

[24] G. 't Hooft, "Computation of the quantum effects due to a four-dimensional pseudoparticle," Physical Review D, vol. 14, no. 12, pp. 3432-3450, 1976.

[25] G. 't Hooft, "Erratum: computation of the quantum effects due to a four-dimensional pseudoparticle (Physical Review D (1978) 18, 6)," Physical Review D, vol. 18, no. 6, pp. 2199-2200, 1978.

[26] M. F. Atiyah, V. G. Drinfeld, N. J. Hitchin, and Y. U. Manin, "Construction of instantons," Physics Letters A, vol. 65, no. 3, pp. 185-187, 1978.

[27] U. Herbst, R. Hofmann, and J. Rohrer, "SU(2) Yang-Mills thermodynamics: two-loop corrections to the pressure," Acta Physica Polonica B, vol. 36, no. 3, 881 pages, 2005.

[28] A. D. Linde, "Infrared problem in the thermodynamics of the Yang-Mills gas," Physics Letters B, vol. 96, no. 3-4, pp. 289-292, 1980.

[29] D. N. Spergel, L. Verde, H. V. Peiris et al., "First-year Wilkinson Microwave Anisotropy Probe (WMAP) observations: determination of cosmological parameters," The Astrophysical Journal Supplement Journal, vol. 148, no. 1, pp. 175-194, 2003.

[30] A. Actor, "Self dual solutions of the temperature SU(2) Yang-Mills theory," Annals of Physics, vol. 148, no. 1 , pp. 32-56, 1983.

[31] A. Chakrabarti, "Periodic generalizations of static, self-dual SU(2) gauge fields," Physical Review D, vol. 35, no. 2, pp. 696-706, 1987. 

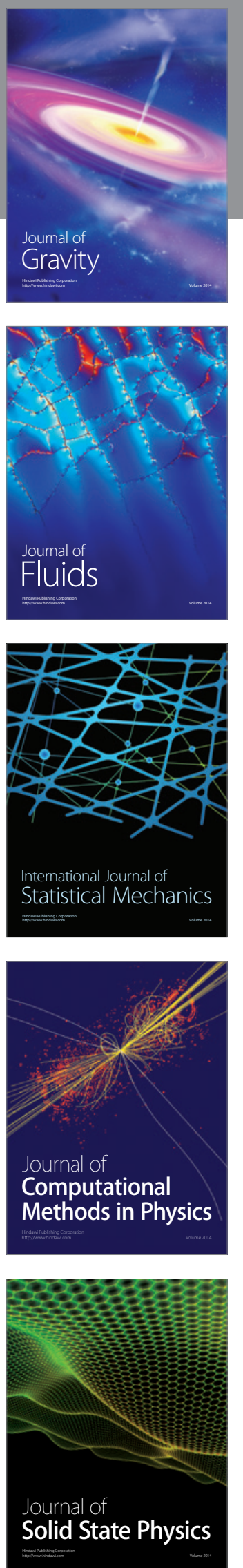
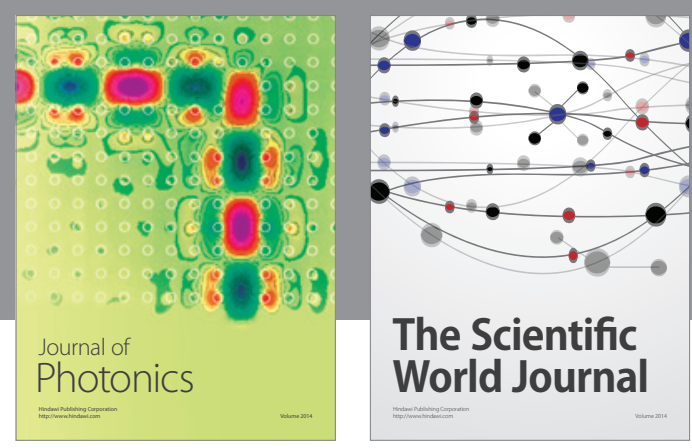

The Scientific World Journal

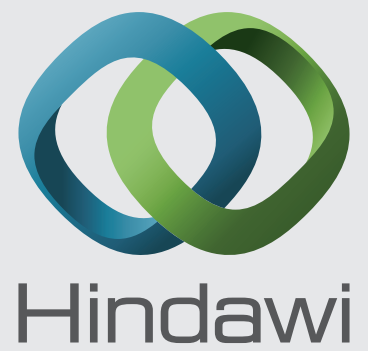

Submit your manuscripts at http://www.hindawi.com
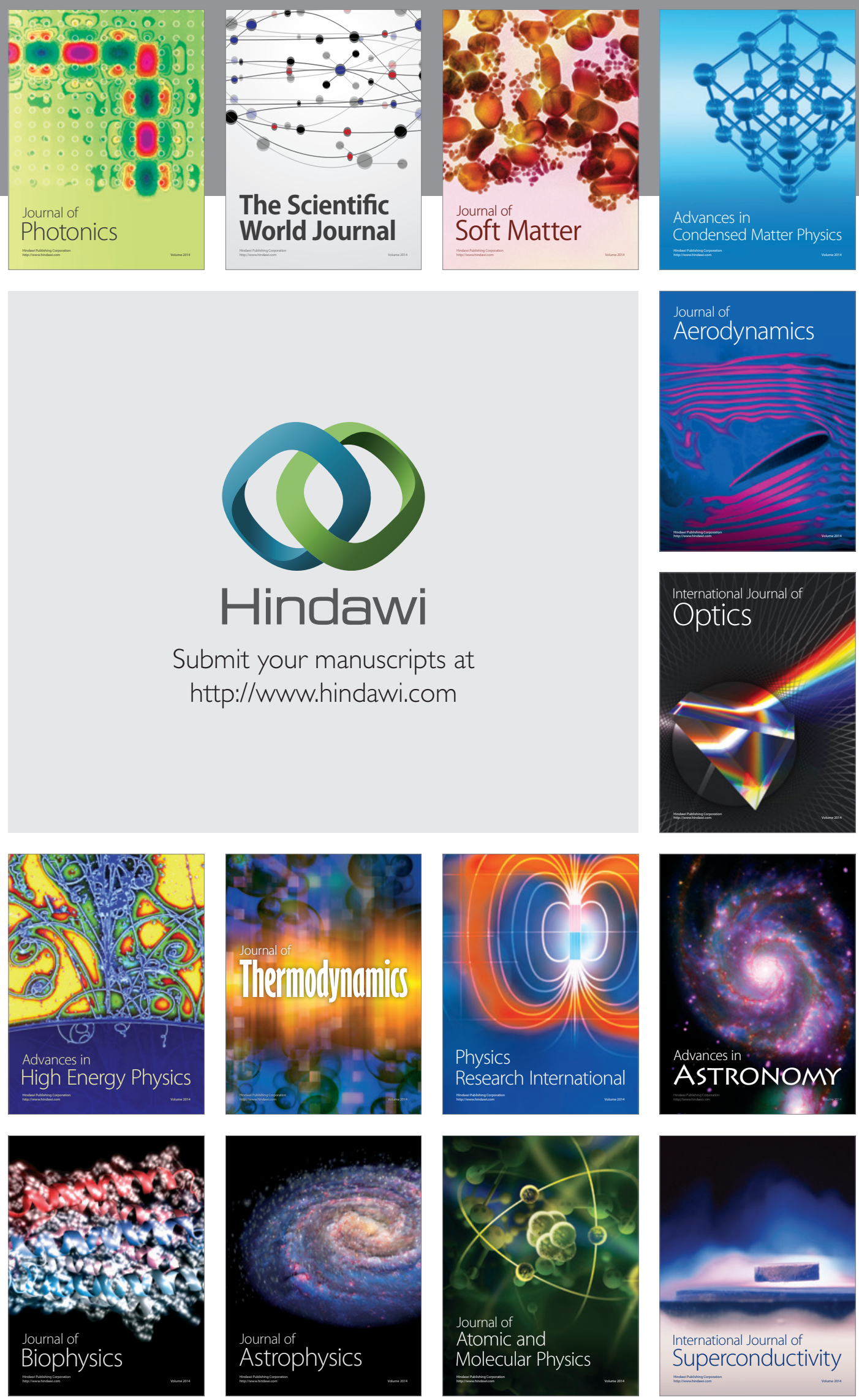
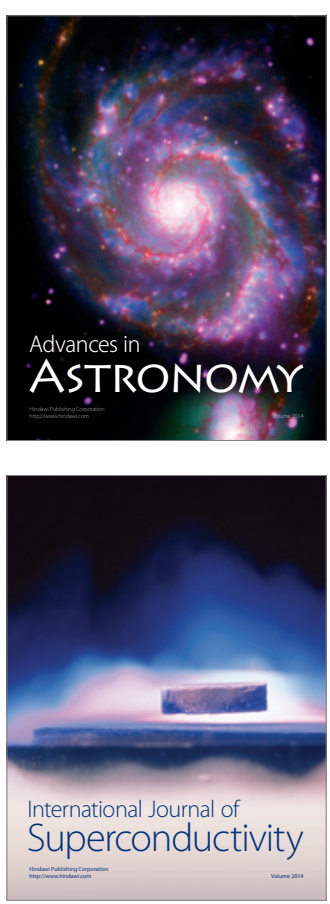